\title{
The factors of personality traits among students of arts, commerce and science of students
}

\author{
Mr. Ankit P. Patel*
}

\section{INTRODUCTION}

Today many interesting researches are being conducted in the field of psychology. This Research on home environment, anxiety, mental health, emotional maturity etc finds the place in many leading journals of psychology. Topic of personality traits has drawn attention of many researchers in psychology. Research in personality traits has received a great deal of attention in the last few decades. Comparative study on personality traits of different social groups is worth exploring. Therefore a researcher has selected an interesting topic of comparing the personality traits of Arts, Commerce and Science students. It is also worthwhile to compare the personality traits of males and females. Different inventories of evaluating personality are available. From the available test of personality, the researcher has selected $16 \mathrm{PF}$ of cattle to collect relevant data. Personality is defined as characteristics and distinctive ways of behaving according to C.T. Morgan. The individual behaves in the environment according to his personality traits. The oldest approach to the problem of identifying characteristic and distinctive ways of behaving is to establish a limited number of traits that can be measured with standardised personality tests. $16 \mathrm{PF}$ is one such standard personality inventory.

A scientific way of selecting and measuring a limited number of traits is the technique of factor analysis. Different researchers have identified several factors of personality and constructed standard personality inventories. One such popular personality test is $16 \mathrm{PF}$ of cattle. The trait name represents the 16 personality factor obtained by factor analysis of large number of rating of different groups. In the present research, researcher has used standardised Gujarati version of original $16 \mathrm{PF}$ of cattle.

*M.A, Clinical Psychology, Sardar Patel University, Vallabh Vidhyanagar, Gujarat 
The International Journal of Indian Psychology: Volume: 01 | Issue: 04 | ISSN 2348-5396 REVIEW OF LITERATURE

A. " A comparative study of factors of personality traits among players of individual and team games of universities of Gujarat" The above research was conducted by Dr. Harish M. Desai, Head of Physical Education, Gujarat University, Ahmadabad, under the guidance of Dr. P.B. Tambar, Director, Department of Physical Education. M. S. University of Baroda. The sample consisted of total 420 players, 210 males and 210 females. Researcher administered $16 \mathrm{PF}$ to those persons. The scoring was done according to manual. The statistical analysis consisted mean, SD and T. Significant difference in personality traits was found in males and females. As well as players of individual events and team events.

B. Personality and culture revisited: Linking traits and dimensions of culture.

The above study was conducted in Tilburg, Netherland, by Greet Hofstede, Robert R Mcnac. It was concerned with trait and preliterate societies. In these research taxonomies of personality traits and cultural values was discussed. The five- factor model of personality is universally valued taxonomy of traits. In analysis of recently reported data, mean personality score of 33 countries were significantly correlated with culture dimension score. Environmental and temperamental explanations were discussed for this.

C. University students personality traits and entrepreneurial intention:

Using entrepreneurship and entrepreneurial attitudes as mediating variable, the study was conducted by Su-Changchen, Professors and Ling-Lingsing, lecturer in National Penghu university of Science \& Technology, Taiwan.

This study aims to probe into relationship between personality traits \& entrepreneurial attitude. A total of 550 questionnaires were distributed to seven universities in Taiwan. After eliminating the questionnaires with incomplete answers \& invalid ones, there were 392 variety samples with a valid return rate of $71.27 \%$. The result indicated that openness to experience; extraversion and conscientiousness influence entrepreneur attitude entrepreneurship \& entrepreneurial attitude reveal partial mediating effects between openness to experience, extraversion and conscientiousness in personality traits \& entrepreneur intention. 
The International Journal of Indian Psychology: Volume: 01 | Issue: 04 | ISSN 2348-5396

OBJECTIVES:

The present research was carried out with the following objectives.

I. To find out the difference in personality traits of Arts, Commerce and Science students.

II. To find out the difference in personality traits between males and females students.

\section{SAMPLE:}

150 students from Lunawada Arts, Commerce and Science College were selected randomly. The distribution of sample is shown as under.

\begin{tabular}{|l|l|l|l|}
\hline Group & Male & Female & Total \\
\hline Arts $\left(\mathrm{P}^{*}\right)$ & 25 & 25 & 50 \\
\hline Commerce $\left(\mathrm{Q}^{* *}\right)$ & 25 & 25 & 50 \\
\hline Science $\left(\mathrm{R}^{* * *}\right)$ & 25 & 25 Interin & 500 nal J Ou11) \\
\hline Grand Total & & $150 \#$ \\
\hline
\end{tabular}

Comparison between these groups will follow the following patterns.

$$
\mathbf{P} * \mathbf{X Q} \mathbf{Q}^{* *}, \mathbf{Q} * \mathbf{X} \mathbf{R} * * *, \mathbf{P} * \mathbf{X} \mathbf{R} * * *
$$

\section{VARIABLES:}

Variables of this study are shown in following table.

Independent variables: - Students of Arts, Commerce \& Science

Dependent variables: - Personality traits

\section{HYPOTHESIS:}

For present study, following hypothesis were framed 


\section{The International Journal of Indian Psychology: Volume: 01 | Issue: 04 | ISSN 2348-5396}

1) There will be no significant difference in factors of personality traits among Arts and Commerce students.

2) There will be no significant difference in factors of personality traits among Commerce and Science students.

3) There will be no significant difference in factors of personality traits among Arts and Science students.

4) There will be no significant difference in factors of personality traits among male and female students.

\section{TOOLS FOR THE DATA COLLECTION:}

Standardized Gujarati version of original 16 PF prepared by Dr. J.H Shah and Dr. R.S Patel of educational faculty of Gujarat University, was used as a tool of data collection. The reliability and validity of the said test has been worked out by researcher of the present test.

Discussion:- Present research has been under taken to compare the factors of personality traits among Arts \& Commerce students, commerce \& science students, Arts \& science students. For the purpose of this study 50 arts, 50 commerce and 50 science students were selected as sample. Each group consisted of 25 male students and 25 female students. Out of total sample of 150 students, 75 were males and 75 were females. It was hypotheses that there is no significant difference in the personality traits among arts and commerce, commerce and science, arts \& science students, male \& female students.

Standardised Gujarati version of 16 PF test prepared by Dr. J. H. Shah and Dr. R. S. Patel was used for collecting data. Two cardboards \& stencil scoring keys were used. Key-1 covered factors (Traits) A, C, F, H, L, N, Q1 \& Q3. Key-2 covered other factors (traits) B, E, G, I, M, O, Q2 \& Q4. The scores were summed up and row scores were covered into "sten" with the help of norms of Form A and form B.

For the statistical analysis of the collected data mean \& S.D. were worked out initially and then to find out significant difference between the groups " $t$ " ratio was worked out. The level of significance for's' ratio was 0.05 . The discussion of four tables is as under:

\section{Discussion on table no 1}

Difference on personality tarts of factors A to Q4 among Arts and Commerce students.
(C) 2014 www.ijip.in
July-September 2014
$19 \mid \mathrm{P}$ a g e 
The International Journal of Indian Psychology: Volume: 01 | Issue: 04 | ISSN 2348-5396

Table no: 1.1 Arts X commerce

\begin{tabular}{|l|l|l|l|l|l|l|l|}
\hline Factor & Group & Mean & SD & SEM & t & level & $\mathrm{N}$ \\
\hline \multirow{2}{*}{$\mathrm{A}$} & Arts & 4.84 & 1.73 & 0.24 & \multirow{2}{*}{1.1686} & & 50 \\
\cline { 2 - 6 } \cline { 7 - 8 } & Commerce & 4.44 & 1.69 & 0.24 & & ns & 50 \\
\hline
\end{tabular}

Table no: 1.2 Arts X commerce

\begin{tabular}{|l|l|l|l|l|l|l|l|}
\hline Factor & Group & Mean & SD & SEM & t & level & N \\
\hline \multirow{2}{*}{ B } & Arts & 4.80 & 2.28 & 0.32 & 0.2161 & & 50 \\
\cline { 2 - 6 } \cline { 7 - 9 } & Commerce & 4.90 & 2.53 & 0.33 & & Ns & 50 \\
\hline
\end{tabular}

Table no: 1.3 Arts X commerce

\begin{tabular}{|l|l|l|l|l|l|l|l|}
\hline Factor & Group & Mean & SD & SEM & t & level & N \\
\cline { 1 - 3 } C & Arts & 3.72 & 1.86 & 0.26 & \multirow{2}{*}{1.7218} & ns & 50 \\
\cline { 2 - 4 } \cline { 7 - 8 } & Commerce & 4.44 & 2.30 & 0.32 & & & 50 \\
\hline
\end{tabular}

Table no: 1.4 Arts X commerce

\begin{tabular}{|l|l|l|l|l|l|l|l|}
\hline Factor & Group & Mean & SD & SEM & t & level & N \\
\hline \multirow{2}{*}{$\mathrm{E}$} & Arts & 5.24 & 1.87 & 0.26 & \multirow{2}{*}{1.8653} & $\mathrm{~ns}$ & 50 \\
\cline { 2 - 4 } \cline { 7 - 8 } & Commerce & 5.96 & 1.99 & 0.28 & & & 50 \\
\hline
\end{tabular}

Table no: 1.5 Arts X commerce

\begin{tabular}{|c|c|c|c|c|c|c|c|}
\hline Factor & Group & Mean & SD & SEM & $\mathrm{t}$ & level & $\mathrm{N}$ \\
\hline \multirow[t]{2}{*}{$\mathrm{F}$} & Arts & 5.58 & 1.77 & 0.25 & 1.0103 & & $50 \mathrm{f}$ \\
\hline & Commerce & 5.26 & 1.37 & 0.19 & & & 50 \\
\hline
\end{tabular}

Table no: 1.6 Arts X commerce

\begin{tabular}{|l|l|l|l|l|l|l|l|}
\hline Factor & Group & Mean & SD & SEM & t & level & $\mathrm{N}$ \\
\hline \multirow{2}{*}{$\mathrm{G}$} & Arts & 4.06 & 1.48 & 0.21 & 0.8695 & & 50 \\
\cline { 2 - 4 } \cline { 7 - 8 } & Commerce & 4.34 & 1.73 & 0.25 & & ns & 50 \\
\hline
\end{tabular}

Table no: 1.7 Arts X commerce

\begin{tabular}{|l|l|l|l|l|l|l|l|}
\hline Factor & Group & Mean & SD & SEM & t & level & N \\
\hline \multirow{2}{*}{$\mathrm{H}$} & Arts & 4.06 & 1.80 & 0.25 & \multirow{2}{*}{0.9253} & & 50 \\
\cline { 2 - 4 } \cline { 7 - 8 } & Commerce & 4.40 & 1.87 & 0.26 & & NS & 50 \\
\hline
\end{tabular}

Table no: 1.8 Arts X commerce

\begin{tabular}{|l|l|l|l|l|l|l|l|}
\hline Factor & Group & Mean & SD & SEM & t & level & N \\
\hline \multirow{2}{*}{ I } & Arts & 4.34 & 2.07 & 0.29 & 0.6300 & NS & 50 \\
\cline { 2 - 4 } \cline { 2 - 4 } & Commerce & 4.60 & 2.06 & 0.29 & & & 50 \\
\hline
\end{tabular}

Table no: 1.9 Arts X commerce

\begin{tabular}{|l|l|l|l|l|l|l|l|}
\hline Factor & Group & Mean & SD & SEM & t & level & N \\
\hline \multirow{2}{*}{ L } & Arts & 5.30 & 1.97 & 0.28 & 0.1636 & & 50 \\
\cline { 2 - 4 } \cline { 7 - 8 } & Commerce & 5.24 & 1.68 & 0.24 & & NS & 50 \\
\hline
\end{tabular}


The International Journal of Indian Psychology: Volume: 01 | Issue: 04 | ISSN 2348-5396

Table no: 1.10 Arts X commerce

\begin{tabular}{|l|l|l|l|l|l|l|l|}
\hline Factor & Group & Mean & SD & SEM & t & level & N \\
\hline \multirow{2}{*}{$\mathrm{M}$} & Arts & 4.82 & 2.62 & 0.37 & \multirow{2}{*}{0.0000} & $\mathrm{NS}$ & 50 \\
\cline { 2 - 4 } \cline { 7 - 8 } & Commerce & 4.82 & 2.62 & 0.37 & & & 50 \\
\hline
\end{tabular}

Table no: 1.11 Arts X commerce

\begin{tabular}{|l|l|l|l|l|l|l|l|}
\hline Factor & Group & Mean & SD & SEM & t & level & N \\
\hline \multirow{2}{*}{$\mathrm{N}$} & Arts & 3.82 & 2.16 & 0.31 & 0.7828 & & 50 \\
\cline { 2 - 4 } \cline { 6 - 8 } & Commerce & 4.14 & 1.92 & 0.27 & & NS & 50 \\
\hline
\end{tabular}

Table no: 1.12 Arts X commerce

\begin{tabular}{|l|l|l|l|l|l|l|l|}
\hline Factor & Group & Mean & SD & SEM & t & level & N \\
\hline \multirow{2}{*}{$\mathrm{O}$} & Arts & 4.20 & 1.77 & 0.25 & \multirow{2}{*}{0.9186} & & 50 \\
\cline { 2 - 4 } \cline { 7 - 8 } & Commerce & 4.56 & 2.13 & 0.30 & & NS & 50 \\
\hline
\end{tabular}

Table no: 1.13 Arts X commerce

\begin{tabular}{|l|l|l|l|l|l|l|l|}
\hline Factor & Group & Mean & SD & SEM & t & level & N \\
\hline \multirow{2}{*}{ Q1 } & Arts & 4.56 & 2.30 & 0.33 & 0.7840 & & 50 \\
\cline { 2 - 4 } \cline { 7 - 8 } & Commerce & 4.90 & 2.02 & 0.29 & & NS & 50 \\
\hline
\end{tabular}

Table no: 14 . Arts X commerce

\begin{tabular}{|l|l|l|l|l|l|l|l|}
\hline Factor & Group & Mean & SD & SEM & t & level & N \\
\hline \multirow{2}{*}{ Q2 } & Arts & 3.94 & 1.65 & 0.23 & 3.3369 & SIG & 50 \\
\cline { 2 - 4 } \cline { 7 - 8 } & Commerce & 5.14 & 1.94 & 0.27 & & & 50 \\
\hline
\end{tabular}

Table no: 1.15 Arts X commerce

\begin{tabular}{|l|l|l|l|l|l|l|l|}
\hline Factor & Group & Mean & SD & SEM & t & level & N \\
\hline \multirow{2}{*}{ Q3 } & Arts & 3.20 & 1.58 & 0.22 & 2.8441 & & 50 \\
\cline { 2 - 6 } & Commerce & 4.30 & 2.23 & 0.32 & & NS & 50 \\
\hline
\end{tabular}

Table no: 1.16 Arts X commerce

\begin{tabular}{|l|l|l|l|l|l|l|l|}
\hline Factor & Group & Mean & SD & SEM & t & level & N \\
\hline \multirow{2}{*}{ Q4 } & Arts & 5.30 & 2.13 & 0.30 & 0.5507 & & 50 \\
\cline { 2 - 4 } \cline { 7 - 8 } & Commerce & 5.06 & 2.23 & 0.31 & & NS & 50 \\
\hline
\end{tabular}

Table No 1 is indicating the difference on personality traits of factors A to Q4 among Arts and commerce students. The difference on each factor is given the columns 1.1, 1.2, 1.3, 1.4, 1.5, 1.6, $1.7,1.8,1.9,1.10,1.11,1.12,1.13,1.14,1.15,1.16 .1 .1$ indicates factor $\mathrm{A}, 1.2$ factor $\mathrm{B}, 1.3$ factor $\mathrm{C}, 1.4$ factor $\mathrm{E}, 1.5$ factor $\mathrm{F}, 1.6$ factor $\mathrm{G}, 1.7$ factor $\mathrm{H}, 1.8$ factor $\mathrm{I}, 1.9$ factor $\mathrm{L}, 1.10$ Factor M, 1.11 Factor N, 1.12 factor O, 1.13 factor Q1, 1.14 factor Q2, 1.15 Factor Q3, 1.16 Factor Q4. 
The International Journal of Indian Psychology: Volume: 01 | Issue: 04 | ISSN 2348-5396

' $t$ ' ratio of each factors is worked out and shown in the above columns. It clearly indicates that' $t$ ' is not significant. Therefore hypothesis No 1 is accepted. Hence it is concluded that there is no significant difference on personality traits of Arts and commerce students.

We can justify this conclusion by saying that there is no much difference in personality traits of Art \& commerce students. The development of personality is not affected by the faculty of education. There are many other environmental factors and heredity which shape our personality.

\section{Chart:}

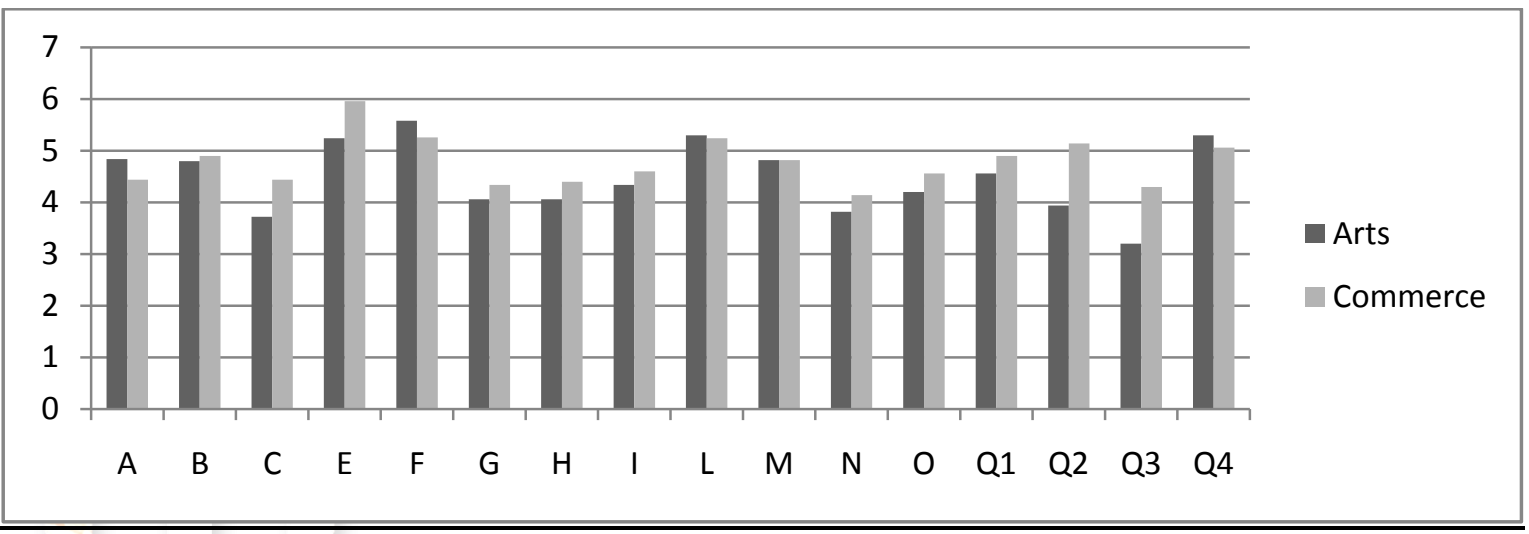

\section{Discussion on Table no 2}

Difference on personality tarts of factors A to Q4 among Commerce and Science students.

Table no 2.1: Commerce X Science

\begin{tabular}{|l|l|l|l|l|l|l|l|}
\hline Factor & Group & Mean & SD & SEM & t & level & N \\
\hline \multirow{2}{*}{ A } & Commerce & 4.44 & 1.73 & 0.24 & \multirow{2}{*}{0.6882} & NS & 50 \\
\cline { 2 - 5 } \cline { 3 - 4 } & Science & 4.18 & 2.07 & 0.29 & & & 50 \\
\hline
\end{tabular}

Table no: 2.2. Commerce and Science

\begin{tabular}{|l|l|l|l|l|l|l|l|}
\hline Factor & Group & Mean & SD & SEM & t & level & N \\
\cline { 1 - 5 } B & Commerce & 4.90 & 2.53 & 0.33 & 1.4141 & NS & 50 \\
\cline { 2 - 4 } & Science & 5.52 & 2.02 & 0.29 & & & 50 \\
\hline
\end{tabular}

Table no: 2.3. Commerce and Science

\begin{tabular}{|l|l|l|l|l|l|l|l|}
\hline Factor & Group & Mean & SD & SEM & t & level & N \\
\hline \multirow{2}{*}{ C } & Commerce & 4.44 & 2.30 & 0.32 & 0.1235 & NS & 50 \\
\cline { 2 - 5 } & Science & 4.49 & 1.66 & 0.24 & & & \\
\cline { 3 - 4 } & &
\end{tabular}

Table no: 2.4 Commerce X Science

\begin{tabular}{|l|l|l|l|l|l|l|l|}
\hline Factor & Group & Mean & SD & SEM & t & level & N \\
\hline \multirow{2}{*}{ E } & Commerce & 5.96 & 1.99 & 0.26 & 1.2888 & NS & 50 \\
\cline { 2 - 4 } \cline { 7 - 8 } & Science & 5.42 & 2.20 & 0.31 & & & 50 \\
\hline
\end{tabular}


The International Journal of Indian Psychology: Volume: 01 | Issue: 04 | ISSN 2348-5396

Table no: 2.5 Commerce X Science

\begin{tabular}{|l|l|l|l|l|l|l|l|}
\hline Factor & Group & Mean & SD & SEM & t & level & N \\
\hline \multirow{2}{*}{ F } & Commerce & 5.26 & 1.37 & 0.19 & 0.2253 & NS & 50 \\
\cline { 2 - 5 } & Science & 5.34 & 2.11 & 0.30 & & & \\
\cline { 3 - 4 } & &
\end{tabular}

Table no: 2.6 Commerce X Science

\begin{tabular}{|l|l|l|l|l|l|l|l|}
\hline Factor & Group & Mean & SD & SEM & t & level & N \\
\hline \multirow{2}{*}{ G } & Commerce & 4.34 & 1.73 & 0.25 & 2.1313 & NS & 50 \\
\cline { 2 - 4 } & Science & 3.60 & 1.69 & 0.24 & & & 50 \\
\hline
\end{tabular}

Table no: 2.7 Commerce X Science

\begin{tabular}{|l|l|l|l|l|l|l|l|}
\hline Factor & Group & Mean & SD & SEM & t & level & N \\
\hline \multirow{2}{*}{$\mathrm{H}$} & Commerce & 4.40 & 1.87 & 0.26 & 0.3248 & NS & 50 \\
\cline { 2 - 5 } \cline { 3 - 4 } & Science & 4.52 & 1.82 & 0.26 & & & 50 \\
\hline
\end{tabular}

Table no: 2.8 Commerce X Science

\begin{tabular}{|l|l|l|l|l|l|l|l|}
\hline Factor & Group & Mean & SD & SEM & t & level & N \\
\hline \multirow{2}{*}{ I } & Commerce & 4.60 & 2.06 & 0.29 & 0.6183 & NS & 50 \\
\cline { 2 - 5 } & Science & 4.34 & 2.14 & 0.30 & & & 50 \\
\hline
\end{tabular}

Table no: 2.9 Commerce X Science

\begin{tabular}{|l|l|l|l|l|l|l|l|}
\hline Factor & Group & Mean & SD & SEM & t & level & N \\
\hline \multirow{2}{*}{ L } & Commerce & 5.24 & 1.68 & 0.24 & 0.3426 & NS & 50 \\
\cline { 2 - 5 } & Science & 5.36 & 1.82 & 0.26 & & & 50 \\
\hline
\end{tabular}

Table no: 2.10 Commerce X Science

\begin{tabular}{|l|l|l|l|l|l|l|l|}
\hline Factor & Group & Mean & SD & SEM & t & level & N \\
\hline \multirow{2}{*}{$\mathrm{M}$} & Commerce & 4.82 & 2.62 & 0.37 & 0.6709 & NS & 50 \\
\cline { 2 - 5 } \cline { 3 - 4 } & Science & 4.52 & 2.41 & 0.34 & & & 50 \\
\hline
\end{tabular}

Table no: 2.11 Commerce X Science

\begin{tabular}{|l|l|l|l|l|l|l|l|}
\hline Factor & Group & Mean & SD & SEM & t & level & N \\
\cline { 1 - 5 } $\mathrm{N}$ & Commerce & 4.14 & 1.92 & 0.27 & \multirow{2}{*}{1.2777} & NS & 50 \\
\cline { 2 - 4 } \cline { 3 - 4 } & Science & 3.62 & 2.15 & 0.30 & & & 50 \\
\hline
\end{tabular}

Table no: 2.12Commerce X Science

\begin{tabular}{|l|l|l|l|l|l|l|l|}
\hline Factor & Group & Mean & SD & SEM & t & level & N \\
\hline \multirow{2}{*}{$\mathrm{O}$} & Commerce & 4.56 & 2.13 & 0.30 & 2.2793 & NS & 50 \\
\cline { 2 - 5 } \cline { 3 - 5 } & Science & 3.68 & 1.71 & 0.24 & & & 50 \\
\hline
\end{tabular}

Table no: 2.13 Commerce X Science

\begin{tabular}{|l|l|l|l|l|l|l|l|}
\hline Factor & Group & Mean & SD & SEM & t & level & N \\
\hline \multirow{2}{*}{ Q1 } & Commerce & 4.90 & 2.02 & 0.29 & 2.0755 & NS & 50 \\
\cline { 2 - 5 } & Science & 4.06 & 2.02 & 0.29 & & & 50 \\
\hline
\end{tabular}


The International Journal of Indian Psychology: Volume: 01 | Issue: 04 | ISSN 2348-5396

Table no: 2.14 Commerce X Science

\begin{tabular}{|l|l|l|l|l|l|l|l|}
\hline Factor & Group & Mean & SD & SEM & t & level & N \\
\hline \multirow{2}{*}{ Q2 } & Commerce & 5.14 & 1.94 & 0.27 & \multirow{2}{*}{3.1691} & NS & 50 \\
\cline { 2 - 5 } & Science & 3.88 & 2.04 & 0.29 & & & \\
\cline { 3 - 4 } & &
\end{tabular}

Table no: 2.15 Commerce X Science

\begin{tabular}{|l|l|l|l|l|l|l|l|}
\hline Factor & Group & Mean & SD & SEM & t & level & N \\
\hline \multirow{2}{*}{ Q3 } & Commerce & 4.30 & 2.23 & 0.32 & 3.7756 & NS & 50 \\
\cline { 2 - 5 } & Science & 2.78 & 1.76 & 0.25 & & & 50 \\
\hline
\end{tabular}

Table no: 2.16 Commerce X Science

\begin{tabular}{|l|l|l|l|l|l|l|l|}
\hline Factor & Group & Mean & SD & SEM & t & level & N \\
\hline \multirow{2}{*}{ Q4 } & Commerce & 5.06 & 2.23 & 0.13 & \multirow{2}{*}{0.4365} & NS & 50 \\
\cline { 2 - 4 } \cline { 2 - 4 } & Science & 5.26 & 2.35 & 0.33 & & & \\
\hline
\end{tabular}

Table no 2 indicates the difference on personality factors (Traits) A to Q4 among commerce \& science students. The difference in each factor is given in the columns 2.1, 2.2, 2.3, 2.4, 2.5, 2.6, 2.7, 2.8, 2.9, 2.10, 2.11, 2.12, 2.13, 2.14, 2.15, 2.16 .

The figures in column 2.1 shows the difference in factor A, 2.2 factor B, 2.3 factor C, 2.4 factor E, 2.5 factor $\mathrm{F}, 2.6$ factor $\mathrm{G}, 2.7$ factor $\mathrm{H}, 2.8$ factor I, 2.9 factor $\mathrm{L}, 2.10$ factor $\mathrm{M}, 2.11$ factor $\mathrm{N}$, 2.12 factor O, 2.13 factor Q1, 2.14 factor Q2, 2.15 factor Q3, 2.16 factor Q4.

' $\mathrm{t}$ ' ratio of each factors is worked out and shown in the above columns. It clearly indicates that ' $\mathrm{t}$ ' is not significant. Therefore hypothesis No 2 is accepted. Hence it is concluded that there is no significant difference on personality traits of commerce and science students.

Commerce and science students do not differ on personality traits as the development of personality traits is not determined by the faculty of education of commerce \& science. The determinants of personality are heredity environment and multiple other factors. 
The International Journal of Indian Psychology: Volume: 01 | Issue: 04 | ISSN 2348-5396

\section{CHART:}

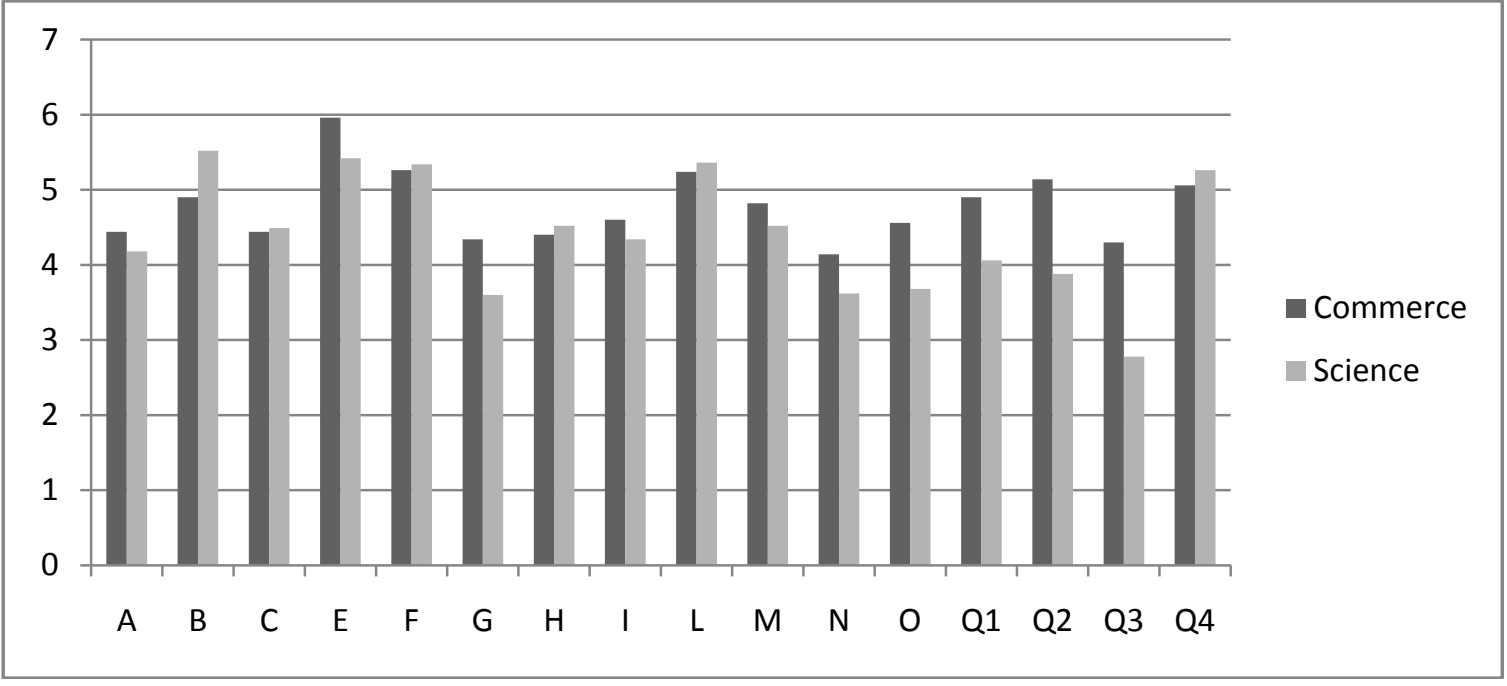

\section{Discussion on Table No 3}

Difference on personality tarts of factors A to Q4 among Arts and Science students.

Table no: 3.1 Arts X Science

\begin{tabular}{|c|c|c|c|c|c|c|c|}
\hline Factor & Group & Mean & SD & SEM & $\mathrm{t}$ & level & $\mathrm{N}$ \\
\hline \multirow[t]{2}{*}{$\mathrm{A}$} & Arts & 4.84 & $1.73 \mathrm{e}$ & 0.2417 & \multirow[t]{2}{*}{$1.7312 a$} & \multirow[t]{2}{*}{ NS Ulin } & 500 \\
\hline & Science & 5.26 & 2.35 & 0.33 & & & 50 \\
\hline
\end{tabular}

Table no: 3.2 Arts X Science

\begin{tabular}{|l|l|l|l|l|l|l|l|}
\hline Factor & Group & Mean & SD & SEM & t & level & N \\
\hline B & Arts & 4.80 & 2.28 & 0.32 & 1.6717 & NS & 50 \\
\cline { 2 - 6 } & Science & 5.52 & 2.02 & 0.29 & & & 50 \\
\hline
\end{tabular}

Table no: 2.3 Arts X Science

\begin{tabular}{|l|l|l|l|l|l|l|l|}
\hline Factor & Group & Mean & SD & SEM & t & level & N \\
\hline C & Arts & 3.72 & 1.86 & 0.26 & 2.1692 & NS & 50 \\
\cline { 2 - 4 } \cline { 7 - 8 } & Science & 4.49 & 1.66 & 0.24 & & & 50 \\
\hline
\end{tabular}

Table no: 3.4 Arts X Science

\begin{tabular}{|l|l|l|l|l|l|l|l|}
\hline Factor & Group & Mean & SD & SEM & t & level & N \\
\hline E & Arts & 5.24 & 1.87 & 0.26 & 0.4415 & NS & 50 \\
\cline { 2 - 4 } & Science & 5.42 & 2.20 & 0.31 & & & 50 \\
\hline
\end{tabular}

Table no: 3.5 Arts X Science

\begin{tabular}{|l|l|l|l|l|l|l|l|}
\hline Factor & Group & Mean & SD & SEM & t & level & N \\
\hline F & Arts & 5.58 & 1.77 & 0.25 & 0.6164 & NS & 50 \\
\cline { 2 - 4 } \cline { 7 - 8 } & Science & 5.34 & 2.11 & 0.30 & & & 50 \\
\hline
\end{tabular}


The International Journal of Indian Psychology: Volume: 01 | Issue: 04 | ISSN 2348-5396

Table no: 3.6 Arts X Science

\begin{tabular}{|l|l|l|l|l|l|l|l|}
\hline Factor & Group & Mean & SD & SEM & t & level & N \\
\hline \multirow{2}{*}{ G } & Arts & 4.06 & 1.48 & 0.21 & \multirow{2}{*}{1.4493} & NS & 50 \\
\cline { 2 - 5 } & Science & 3.60 & 1.69 & 0.24 & & & \\
\cline { 7 - 8 } & &
\end{tabular}

Table no: 3.7 Arts X Science

\begin{tabular}{|l|l|l|l|l|l|l|l|}
\hline Factor & Group & Mean & SD & SEM & t & level & N \\
\cline { 1 - 5 } H & Arts & 4.06 & 1.80 & 0.25 & \multirow{2}{*}{1.2702} & NS & 50 \\
\cline { 2 - 4 } & Science & 4.52 & 1.82 & 0.26 & & & 50 \\
\hline
\end{tabular}

Table no: 3.8 Arts X Science

\begin{tabular}{|l|l|l|l|l|l|l|l|}
\hline Factor & Group & Mean & SD & SEM & t & level & N \\
\hline \multirow{2}{*}{ I } & Arts & 4.34 & 2.07 & 0.29 & \multirow{2}{*}{0.0000} & NS & 50 \\
\cline { 2 - 5 } & Science & 4.34 & 2.14 & 0.30 & & & \\
\cline { 3 - 4 } & &
\end{tabular}

Table no: 3.9 Arts X Science

\begin{tabular}{|l|l|l|l|l|l|l|l|}
\hline Factor & Group & Mean & SD & SEM & t & level & N \\
\hline \multirow{2}{*}{ L } & Arts & 5.30 & 1.97 & 0.28 & 0.1583 & NS & 50 \\
\cline { 2 - 4 } \cline { 2 - 4 } & Science & 5.36 & 1.82 & 0.26 & & & 50 \\
\hline
\end{tabular}

Table no: 3.10 Arts X Science

\begin{tabular}{|l|l|l|l|l|l|l|l|}
\hline Factor & Group & Mean & SD & SEM & t & level & N \\
\hline \multirow{2}{*}{ M } & Arts & 4.82 & 2.62 & 0.37 & 0.5964 & NS & 50 \\
\cline { 2 - 5 } & Science & 4.52 & 2.41 & 0.34 & & & 50 \\
\hline
\end{tabular}

Table no: 3.11 Arts X Science

\begin{tabular}{|l|l|l|l|l|l|l|l|}
\hline Factor & Group & Mean & SD & SEM & t & level & N \\
\hline \multirow{2}{*}{$\mathrm{N}$} & Arts & 3.82 & 2.16 & 0.31 & 0.4640 & NS & 50 \\
\cline { 2 - 5 } & Science & 3.62 & 2.15 & 0.30 & & & 50 \\
\hline
\end{tabular}

Table no: 3.12 Arts X Science

\begin{tabular}{|l|l|l|l|l|l|l|l|}
\hline Factor & Group & Mean & SD & SEM & t & level & N \\
\hline \multirow{2}{*}{$\mathrm{O}$} & Arts & 4.20 & 1.77 & 0.25 & \multirow{2}{*}{1.4938} & NS & 50 \\
\cline { 2 - 5 } & Science & 3.68 & 1.71 & 0.24 & & & 50 \\
\hline
\end{tabular}

Table no: 3.13 Arts X Science

\begin{tabular}{|l|l|l|l|l|l|l|l|}
\hline Factor & Group & Mean & SD & SEM & t & level & N \\
\hline \multirow{2}{*}{ Q1 } & Arts & 4.56 & 2.30 & 0.33 & 1.1525 & NS & 50 \\
\cline { 2 - 4 } & Science & 4.06 & 2.02 & 0.29 & & & 50 \\
\hline
\end{tabular}

Table no: 3.14 Arts X Science

\begin{tabular}{|l|l|l|l|l|l|l|l|}
\hline Factor & Group & Mean & SD & SEM & t & level & N \\
\hline \multirow{2}{*}{ Q2 } & Arts & 3.94 & 1.65 & 0.23 & \multirow{2}{*}{0.1620} & NS & 50 \\
\cline { 2 - 4 } & Science & 3.88 & 2.04 & 0.29 & & & 50 \\
\hline
\end{tabular}


The International Journal of Indian Psychology: Volume: 01 | Issue: 04 | ISSN 2348-5396

Table no: 3.15 Arts X Science

\begin{tabular}{|l|l|l|l|l|l|l|l|}
\hline Factor & Group & Mean & SD & SEM & t & level & N \\
\hline \multirow{2}{*}{ Q3 } & Arts & 3.20 & 1.58 & 0.22 & \multirow{2}{*}{1.2546} & NS & 50 \\
\cline { 2 - 4 } \cline { 3 - 4 } & Science & 2.78 & 1.76 & 0.25 & & & 50 \\
\hline
\end{tabular}

Table no: 3.16 Arts X Science

\begin{tabular}{|l|l|l|l|l|l|l|l|}
\hline Factor & Group & Mean & SD & SEM & t & level & N \\
\hline \multirow{2}{*}{ Q4 } & Arts & 5.30 & 2.13 & 0.30 & 0.0891 & NS & 50 \\
\cline { 2 - 5 } & Science & 5.26 & 2.35 & 0.33 & & & 50 \\
\hline
\end{tabular}

Table no 3 indicates the difference on personality factors (Traits) A to Q4 among arts \& science students. The difference in each factor is given in the columns 3.1, 3.2, 3.3, 3.4, 3.5, 3.6, 3.7, 3.8, $3.9,3.10,3.11,3.12,3.13,3.14,3.15,3.16$.

The figures in column 3.1 shows the difference in factor A, 3.2 factor B, 3.3 factor C, 3.4 factor E, 3.5 factor $F, 3.6$ factor $G, 3.7$ factor $H, 3.8$ factor I, 3.9 factor $L, 3.10$ factor $M, 3.11$ factor $\mathrm{N}$, 3.12 factor O, 3.13 factor Q1, 3.14 factor Q2, 3.15 factor Q3, 3.16 factor Q4.

' $t$ ' ratio of each factors is worked out and shown in the above columns. It clearly indicates that ' $t$ ' is not significant at 0.05 level. Therefore hypothesis No 3 is accepted. Hence it is concluded that there is no significant difference on personality traits among of arts and science students.

The result shows that the arts \& science students do not differ significantly on personality traits. The reason for this result is that personality traits are not determined by the faculty of education of art \& science students. The determinants of personality are heredity, environment and multiple other factors.

Chart:

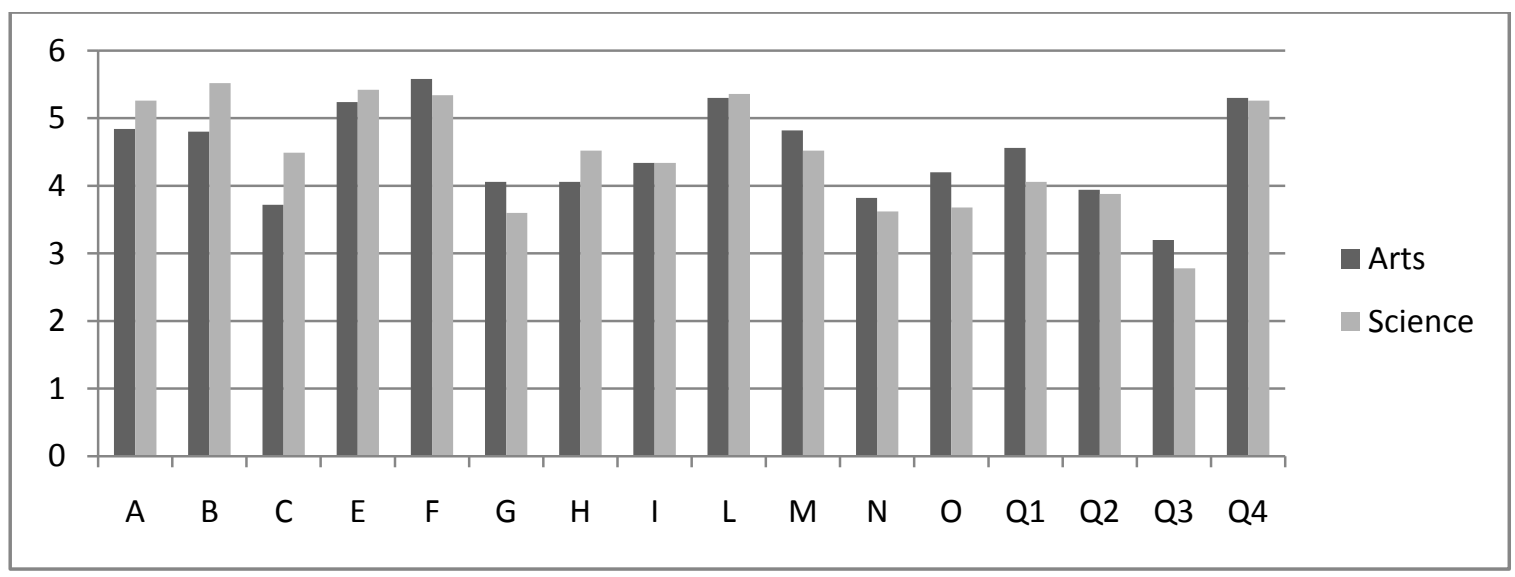


The International Journal of Indian Psychology: Volume: 01 | Issue: 04 | ISSN 2348-5396

\section{Discussion on Table No 4}

Difference on personality tarts of factors A to Q4. among Male and Female students.

Table no: 4.1 Male X Female

\begin{tabular}{|l|l|l|l|l|l|l|l|}
\hline Factor & Group & Mean & SD & SEM & t & level & N \\
\hline \multirow{2}{*}{ A } & Male & 4.85 & 1.92 & 0.22 & 2.4748 & NS & 75 \\
\cline { 2 - 4 } \cline { 7 - 8 } & Female & 4.12 & 1.70 & 0.20 & & & 75 \\
\hline
\end{tabular}

Table no: 4.2 Male X Female

\begin{tabular}{|l|l|l|l|l|l|l|l|}
\hline Factor & Group & Mean & SD & SEM & t & level & N \\
\hline \multirow{2}{*}{ B } & Male & 5.04 & 2.18 & 0.25 & 0.1826 & NS & 75 \\
\cline { 2 - 5 } \cline { 2 - 4 } & Female & 5.11 & 2.29 & 0.26 & & & 75 \\
\hline
\end{tabular}

Table no: 4.3 Male X Female

\begin{tabular}{|l|l|l|l|l|l|l|l|}
\hline Factor & Group & Mean & SD & SEM & t & level & N \\
\cline { 1 - 5 } C & Male & 4.49 & 1.98 & 0.23 & \multirow{2}{*}{1.7424} & NS & 75 \\
\cline { 2 - 5 } \cline { 3 - 4 } & Female & 3.93 & 1.95 & 0.23 & & & 75 \\
\hline
\end{tabular}

Table no: 4.4 Male X Female

\begin{tabular}{|l|l|l|l|l|l|l|l|}
\hline Factor & Group & Mean & SD & SEM & t & level & N \\
\hline \multirow{2}{*}{ E } & Male & 5.27 & 1.83 & 0.21 & \multirow{2}{*}{1.6570} & NS & 75 \\
\cline { 2 - 4 } \cline { 3 - 5 } & Female & 5.81 & 2.20 & 0.25 & & & 75 \\
\hline
\end{tabular}

Table no: 4.5 Male X Female

\begin{tabular}{|c|c|c|c|c|c|c|c|}
\hline Factor & Group & Mean & SD & SEM & $\mathrm{t}$ & level & $\mathrm{N}$ \\
\hline \multirow[t]{2}{*}{$\mathrm{F}$} & Male & 5.45 & 1.92 & 0.22 & \multirow{2}{*}{0.4145} & \multirow[t]{2}{*}{ NS } & 75 \\
\hline & Female & 5.33 & 1.61 & 0.19 & & & 75 \\
\hline
\end{tabular}

Table no: 4.6 Male X Female

\begin{tabular}{|l|l|l|l|l|l|l|l|}
\hline Factor & Group & Mean & SD & SEM & t & level & N \\
\hline \multirow{2}{*}{ G } & Male & 4.21 & 1.61 & 0.19 & 1.5870 & NS & 75 \\
\cline { 2 - 4 } \cline { 7 - 8 } & Female & 3.79 & 1.68 & 0.19 & & & 75 \\
\hline
\end{tabular}

Table no: 4.7 Male X Female

\begin{tabular}{|l|l|l|l|l|l|l|l|}
\hline Factor & Group & Mean & SD & SEM & t & level & N \\
\hline \multirow{2}{*}{$\mathrm{H}$} & Male & 4.91 & 1.89 & 0.22 & 4.0804 & SIG & 75 \\
\cline { 2 - 5 } \cline { 3 - 5 } & Female & 3.75 & 1.58 & 0.18 & & 0.01 & 75 \\
\hline
\end{tabular}

Table no: 4.8 Male X Female

\begin{tabular}{|l|l|l|l|l|l|l|l|}
\hline Factor & Group & Mean & SD & SEM & t & level & N \\
\hline \multirow{2}{*}{ I } & Male & 4.85 & 1.93 & 0.22 & 2.5585 & NS & 75 \\
\cline { 2 - 4 } \cline { 7 - 8 } & Female & 4.00 & 2.15 & 0.25 & & & 75 \\
\hline
\end{tabular}

Table no: 4.9 Male X Female

\begin{tabular}{|l|l|l|l|l|l|l|l|}
\hline Factor & Group & Mean & SD & SEM & t & level & N \\
\hline \multirow{2}{*}{ L } & Male & 5.23 & 1.67 & 0.19 & 0.4933 & NS & 75 \\
\cline { 2 - 4 } \cline { 7 - 8 } & Female & 5.37 & 1.96 & 0.23 & & & 75 \\
\hline
\end{tabular}

Table no: 4.10 Male X Female

\begin{tabular}{|l|l|l|l|l|l|l|l|}
\hline Factor & Group & Mean & SD & SEM & t & level & N \\
\hline \multirow{2}{*}{ M } & Male & 4.85 & 2.37 & 0.27 & 0.6914 & NS & 75 \\
\cline { 2 - 4 } \cline { 3 - 4 } & Female & 4.59 & 2.35 & 0.27 & & & 75 \\
\hline
\end{tabular}


The International Journal of Indian Psychology: Volume: 01 | Issue: 04 | ISSN 2348-5396

Table no: 4.11 Male X Female

\begin{tabular}{|l|l|l|l|l|l|l|l|}
\hline Factor & Group & Mean & SD & SEM & t & level & N \\
\hline \multirow{2}{*}{$\mathrm{N}$} & Male & 4.47 & 1.89 & 0.22 & \multirow{2}{*}{3.7313} & SIG & 75 \\
\cline { 2 - 4 } \cline { 3 - 4 } & Female & 3.25 & 2.09 & 0.24 & & 0.01 & 75 \\
\hline
\end{tabular}

Table no: 4.12 Male X Female

\begin{tabular}{|l|l|l|l|l|l|l|l|}
\hline Factor & Group & Mean & SD & SEM & t & level & N \\
\cline { 1 - 5 } O & Male & 4.44 & 1.70 & 0.20 & 1.9059 & NS & 75 \\
\cline { 2 - 4 } \cline { 2 - 3 } & Female & 3.85 & 2.05 & 0.24 & & & 75 \\
\hline
\end{tabular}

Table no: 4.13 Male X Female

\begin{tabular}{|l|l|l|l|l|l|l|l|}
\hline Factor & Group & Mean & SD & SEM & t & level & N \\
\hline \multirow{2}{*}{ Q1 } & Male & 4.91 & 1.76 & 0.20 & 2.3278 & NS & 75 \\
\cline { 2 - 4 } \cline { 7 - 8 } & Female & 4.11 & 2.40 & 0.28 & & & 75 \\
\hline
\end{tabular}

Table no: 4.14 Male X Female

\begin{tabular}{|l|l|l|l|l|l|l|l|}
\hline Factor & Group & Mean & SD & SEM & t & level & N \\
\hline \multirow{2}{*}{ Q2 } & Male & 4.77 & 1.71 & 0.20 & 2.9072 & NS & 75 \\
\cline { 2 - 5 } & Female & 3.87 & 2.09 & 0.24 & & & 75 \\
\hline
\end{tabular}

Table no: 4.15 Male X Female

\begin{tabular}{|l|l|l|l|l|l|l|l|}
\hline Factor & Group & Mean & SD & SEM & t & level & N \\
\hline \multirow{2}{*}{ Q3 } & Male & 3.75 & 2.05 & 0.24 & 2.0053 & NS & 75 \\
\cline { 2 - 5 } \cline { 3 - 4 } & Female & 3.11 & 1.86 & 0.21 & & & 75 \\
\hline
\end{tabular}

Table no: 4.16 Male X Female

\begin{tabular}{|l|l|l|l|l|l|l|l|}
\hline Factor & Group & Mean & SD & SEM & t & level & N \\
\hline \multirow{2}{*}{ Q4 } & Male & 5.27 & 2.09 & 0.24 & 0.3291 & NS & 75 \\
\cline { 2 - 5 } & Female & 5.15 & 2.36 & 0.27 & & & 75 \\
\hline
\end{tabular}

Table no 4 indicates the differences on personality factors (Traits) A to Q4 among boys \& girls.

The difference in each factor is given in the columns 4.1, 4.2, 4.3, 4.4, 4.5, 4.6, 4.7, 4.8, 4.9, 4.10, 4.11, 4.12, 4.13, 4.14, 4.15, 4.16.

The figures in column 4.1 shows the difference in factor $\mathrm{A}, 4.2$ factor $\mathrm{B}, 4.3$ factor $\mathrm{C}, 4.4$ factor E, 4.5 factor $F, 4.6$ factor $\mathrm{G}, 4.7$ factor $\mathrm{H}, 4.8$ factor I, 4.9 factor $\mathrm{L}, 4.10$ factor $\mathrm{M}, 4.11$ factor $\mathrm{N}$, 4.12 factor O, 4.13 factor Q1, 4.14 factor Q2, 4.15 factor Q3, 4.16 factor Q4.

' $t$ ' ratio of each factors is worked out and shown in the above columns. The results indicate that except $4.7 \mathrm{H}$ factor and $4.11 \mathrm{~N}$ factor ' $\mathrm{t}$ ' is not significant at .05 level. Therefore it is concluded that except factor $\mathrm{H}$ and Factor $\mathrm{N}$ there is no significant difference in the remaining factors of personality traits of boys and girls. ' $\mathrm{t}$ ' ratio of $\mathrm{H}$ factors and $\mathrm{N}$ factor is significant. Factor $\mathrm{H}$ means "timid" and "venturesome" that is on timidity and venture trait of personality, boys and girls significantly differ. About venture or timidity, there may be differences in boys and girls. It 
The International Journal of Indian Psychology: Volume: 01 | Issue: 04 | ISSN 2348-5396

is quite likely that some girls may be more adventurous than boys. Similarly, the results about factor $\mathrm{N}$ refer to forthright \& shrewd. The girls \& boys differ in this trait.

\section{CHART:}

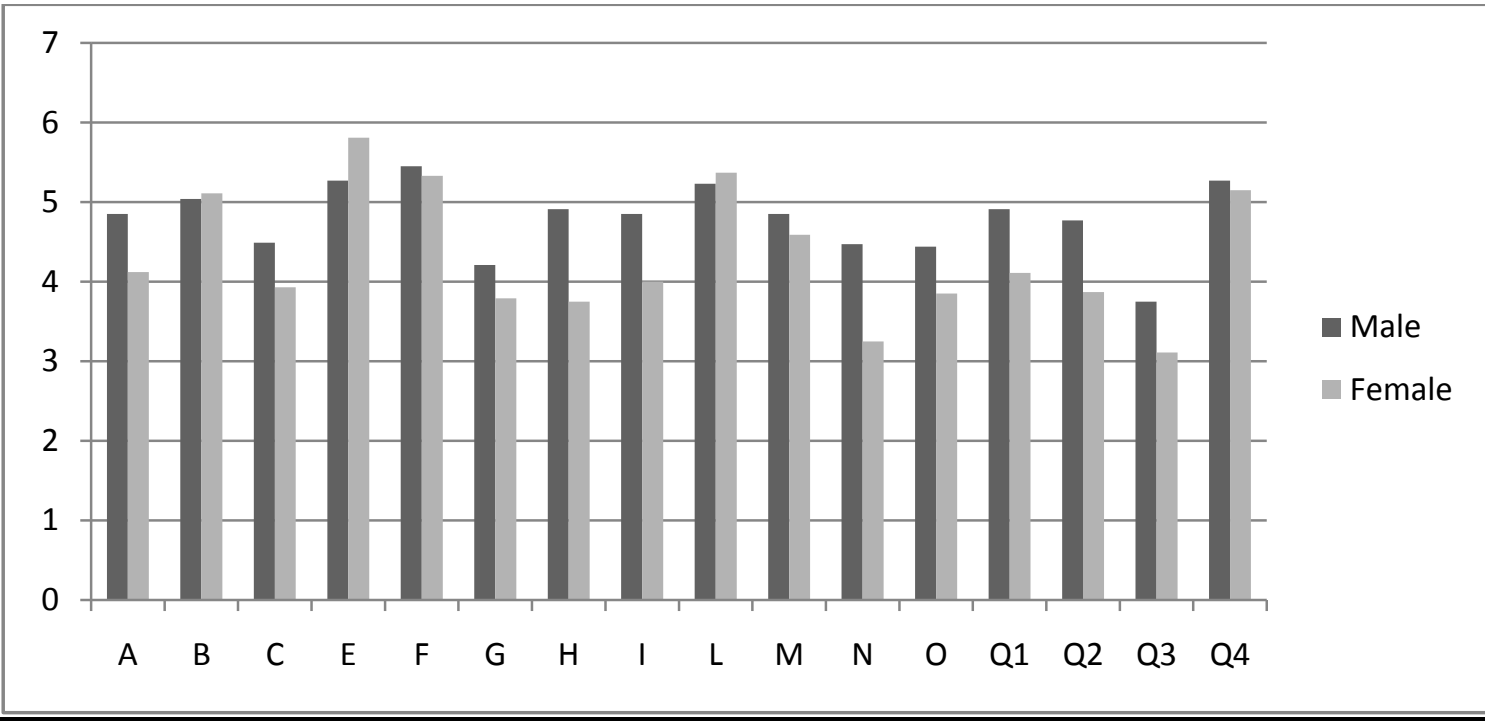

CONCLUSIONS

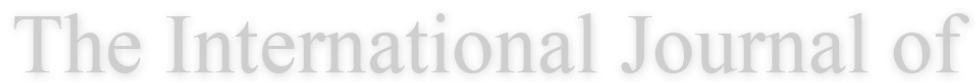

1. There is no significant difference on personality traits of arts and commerce students (' $t$ ' not significant, see table No 1)

2. There is no significant difference on personality traits of commerce and science students (' $\mathrm{t}$ ' not significant, see table No 2)

3. There is no significant difference on personality traits of arts and science students (' $t$ ' not significant, see table No 3)

4. There is no significant difference on personality traits - A, B, C, E, F, G, I, L, M, O, Q1, Q2, Q3, Q4 of boys and girls ('t' for above factors is not significant, see table No 4)

There is a significant difference on personality traits of $\mathrm{H} \& \mathrm{~N}$ of boys and girls ( ' $\mathrm{t}$ ' for these factors is significant, see table No 4) 
The International Journal of Indian Psychology: Volume: 01 | Issue: 04 | ISSN 2348-5396 SUGGESTIONS

1. A research project may be undertaken to compare personality traits of graduates and post graduate students.

2. A similar study can be done with the student of engineering and medical students.

3. A similar study can be performed on international basis.

4. Personality traits can be measured by many other personality inventories

\section{REFERENCES}

1. A comparative study of personality traits among players of individual and team games of universities of Gujarat (Using 16 PF) -Dr. H.M. Desai, Head of Physical education, Gujarat University

2. A guide to the clinical use of $16 \mathrm{PF}-\mathrm{Champaign}$

3. An introduction to the five factor model and its applications -Journals of personality 60 (2) page $175-215$

4. Brief introduction to Psychology - C.T. Morgan

5. Dimensions of personality-Eyesenk

6. Introduction to Psychology -Hilgard and Atikinson

7. Large scale cross validation of personality structure defined by $16 \mathrm{PF}$-Psychological reports 59 (2) $683-693$

8. Personality and culture revisited and Dimensions of culture-Geert Hofstely and others

9. Psychological testing-Anastasi

10. The 16 personality factor questionnaire ( $16 \mathrm{PF}$ ) -Cattle and Mead

11. The big five personality traits, learning styles and academic achievement-Ko marragu \& others

12. University students personality traits and entrepreneurial intentions -Su-chang chen \& others 
The International Journal of Indian Psychology: Volume: 01 | Issue: 04 | ISSN 2348-5396

Annexure-A

Explanation of factors

\begin{tabular}{|l|l|l|l|}
\hline Sr No & Factor & Low score & High score \\
\hline 1 & A & Reserved & Out going \\
\hline 2 & B & Less intelligent & High intelligent \\
\hline 3 & C & Affected by feelings & Emotionally stable \\
\hline 4 & E & Submissive & Dominant \\
\hline 5 & F & Serious & Happy quickly \\
\hline 6 & G & Expedient & Conscientious \\
\hline 7 & H & Timid & Venturesome \\
\hline 8 & I & Tough minded & Sensitive \\
\hline 9 & L & Trusting & Suspicious \\
\hline 10 & M & Practical & Imaginative \\
\hline 11 & N & Forthright & Shrewd \\
\hline 12 & O & Self assured IC IntCIT & Apprehensive \\
\hline 13 & Q1 & Conservative & Experimenting \\
\hline 14 & Q2 & Group dependent & Self sufficient \\
\hline 15 & Q3 & Uncontrolled & Controlled \\
\hline 16 & Q4 & Relaxed & Tense \\
\hline
\end{tabular}

